\title{
Cardiac Findings in 31 Patients with Noonan's Syndrome
}

\author{
Débora Romeo Bertola, Chong A e Kim, Sofia M. M. Sugayama, Lilian Maria José Albano, \\ Jaqueline Wagenführ, Regina Lúcia Moysés, Claudette Hajaj Gonzalez \\ São Paulo, SP - Brazil
}

Objective - To evaluate cardiac findings in 31 Noonan syndrome patients.

Methods - Thirty-one ( 18 males and 13 females)patients from 26 families affected with Noonan's syndrome were evaluated from the cardiac point of view with electrocardiography and echodopplercardiography.

Results - Twenty patients had some type of cardiac abnormality. The most frequent was pulmonary valve stenosis followed by hypertrophic myocardiopathy, commonly associated with valve defects. Upper deviation of the QRS axis was observed in $80 \%$ of these patients.

Conclusion - In view of the high frequency and diversity of cardiac abnormalities present in Noonan syndrome, cardiac evaluation with electrocardiography and echocardiography should be performed in all patients diagnostically suspected of having this disease.

Key words: Noonan syndrome, pulmonary valve stenosis, hypertrophic myocardiopathy .

Unidade de Genética do Instituto da Criança

Faculdade de Medicina da Universidade de São Paulo

Mailing address: Chong Ae Kim - Genética - InCor - Av. Dr. Enéas C. Aguiar, 647

- 05403-900 - São Paulo, SP 
Noonan Syndrome diagnoses of these individuals; were based on the clinical criteria established by van der Burgt et al. ${ }^{7}$. Twenty-six proposed patients and their firstdegree relatives (parents and siblings) were evaluated; recurrence of the disease was observed in five relatives. One of the chosen patients was an adopted child and another's father was not available for evaluation. Of the 29 remaining cases, $8(28 \%)$ were familial and $21(72 \%)$ sporadic. Eighteen patients were males and 13 were females, with ages ranging from 3 months to 41 years (average 12 years). One of the patients had already passed away at the time of the study; his data were obtained from medical files; photographs were used to analyze some of his craniofacial characteristics. The impossibility of detecting some of the craniofacial dysmorphisms in this patient is reflected in the total number of these findings shown (Table I).

The cardiac evaluation was based on a special physical examination, on the electrocardiogram, and on the echocardiogram.

Subjects affected with Noonan's syndrome were only included in the study after signing an informed consent, for themselves when adults, or by legal guardians when underage.

\section{Results}

The most commonly observed clinical findings in patients with Noonan's syndrome were: low stature, craniofacial dysmorphism, short or webbed, cardiac anomalies, sternal deformity, and the presence of pads at the tip of the fingers and toes (Table I).

Chromosome studies showed normal results for all patients.

Twenty (65\%) of the patients had some form of echocardiographic abnormality (Table II).

Signs of right or left ventricular hypertrophy were present in 13 and 5 patients, respectively (Table III).

Upper deviation of the QRS axis was a rather common finding in $80 \%$ of these patients.

\section{Discussion}

Cardiac anomalies are common in Noonan syndrome and the major cause of morbidity and mortality in this disease.

In the present study, $65 \%$ of affected individuals had some form of cardiac abnormality. This frequency is higher than that estimated in other works $(3,8)$, probably because the majority of our patients were sent by the Heart Institute and were known to have a cardiac anomaly, and also because a Genetic Unit of a tertiary hospital, in this case the Children's Institute, tends to receive only the more serious cases.

A cardiac murmur was heard in $12(60 \%)$ of the neonates with cardiac problems, allowing for a precocious, adequate follow-up.

In agreement with literature reports, the most frequent cardiac anomaly was pulmonary stenosis, found in $70 \%$ of

\begin{tabular}{|lc|}
\hline \multicolumn{2}{|c|}{ Table I - Clinical findings in 31 Noonan syndrome } \\
patients
\end{tabular}

\begin{tabular}{|lcc|}
\hline \multicolumn{2}{|c|}{ Table II - Cardiac anomalies observed in Noonan syndrome } \\
patients
\end{tabular}

\begin{tabular}{|c|c|c|}
\hline \multicolumn{3}{|c|}{$\begin{array}{c}\text { Table III - Electrocardiographic findings in Noonan syndrome } \\
\text { patients }\end{array}$} \\
\hline Findings & $\mathrm{N}^{\mathrm{o}}$ & $\%$ \\
\hline RVO & 12 & $57 \%$ \\
\hline LVO & 5 & $24 \%$ \\
\hline RAO & 1 & $5 \%$ \\
\hline RBB & 2 & $9 \%$ \\
\hline Sinus bradycardia & 1 & $5 \%$ \\
\hline Total & 21 & $100 \%$ \\
\hline $\begin{array}{l}\text { RSO- right ventric } \\
\text { atrium overload; } \mathrm{I}\end{array}$ & the & d; RAO-right \\
\hline
\end{tabular}




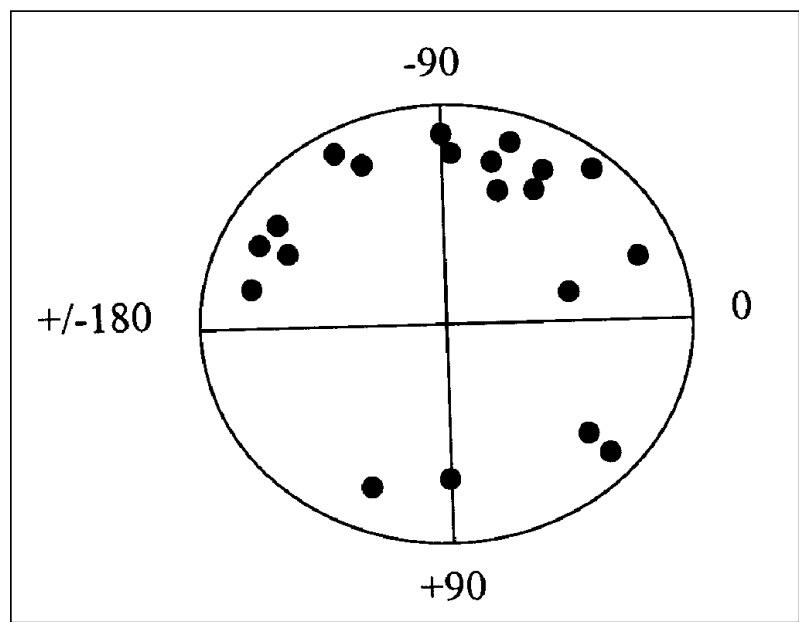

Fig. 1 - QRS axes in patients with cardiac anomalies.

the patients with cardiac problems. The pressure gradient between the right ventricle and the pulmonary arterial trunk varied between 23 and $121 \mathrm{mmHg}$ in these patients. Three (21\%) had a pressor gradient above $60 \mathrm{mmHg}$ characterizing important pulmonary stenosis, 4 (29\%) slight stenosis (pressor gradient between 20 and $40 \mathrm{mmHg}$ ), and $7(50 \%)$ moderate pulmonary stenosis. The most common associated anomalies were interarterial communication and hypertrophic myocardiopathy. Char et al. ${ }^{9}$, studying 45 cases of Noonan' syndrome, observed interarterial communication to be the most frequent cardiac defect associated with pulmonary stenosis.

Echocardiographic findings showed a dysplasic pulmonary valve in 4 (24\%) of our cases of pulmonary valve stenosis. Burch et al. ${ }^{4}$, in an echocardiographic study of 119 Noonan syndrome patients, diagnosed dysplasic valves in $8(27 \%)$ of 30 of these patients- who also had pulmonary stenosis.

Despite the presence of pulmonary valve dysplasia in one third of the patients, the majority of them had no evidence of dysplasia on echocardiography; this observation should be considered in the therapy of these individuals.

Percutaneous dilatation with a balloon catheter, the treatment of choice in cases of moderate and serious pulmonary stenosis, is rarely effective when the valve is dysplasic ${ }^{5}$. Ishizawa et al. ${ }^{10}$, who performed this procedure in 4 Noonan syndrome patients with dysplasic pulmonary valves, obtained good results in two of them. The authors postulated that valvuloplasty should be performed as an initial procedure in patients with Noonan syndrome and pulmonary stenosis, even when evidence of valve dysplasia is found. In our study, three patients, one with serious, two with moderate pulmonary stenosis, respectively, who underwent valvuloplasty, had a significant reduction in the pressor gradient of those with moderate stenosis. It is important to point out that none of these three patients had signs of valve dysplasia on the echocardiogram. Four patients with Noonan syndrome underwent surgical correction of pulmonary stenosis (commissurotomy, pulmonary valvuloplasty, myectomy, and amplification of the right ventricle's outlet by insertion of a bovine pericardium graft), of these two had a pressor gradient above $110 \mathrm{mmHg}$, one had moderate pulmonary stenosis, and in one, no reduction of in the pressor gradient was observed following valvuloplasty.

The second most common cardiac anomaly of Noonan's syndrome is hypertrophic myocardiopathy, also observed in our study. Differently from the non-syndrome form, Noonan syndrome hypertrophic myocardiopathies are frequently associated with a valve anomaly, mainly pulmonary stenosis. Four of our patients with hypertrophic myocardiopathy also had involvement of pulmonary, aortic, or mitral valves.

Interventricular communication and aortic valve thickening were other cardiac findings diagnosed with echocardiography in our study.

The electrocardiogram showed an upper deviation of the QRS axis in $16(60 \%)$ patients with cardiac abnormalities. This was observed in cases of pulmonary valve stenosis, supravalvar pulmonary stenosis, hypertrophic myocardiopathy, and interventricular communication. However, it was not found in patients affected by Noonan's syndrome without cardiac anomalies. Neither was there a direct correlation between the degree of obstruction of the right ventricle's outlet and axis deviation. The physiopathology of this symptom is not fully known, but appears to involve not only counterclockwise rotation of the heart, but also a disturbance in the conduction system of affected patients ${ }^{11}$. This electrocardiographic finding may aid in the diagnosis of Noonan syndrome.

Cardiac involvement is rather frequent in Noonan syndrome patients, with peculiarities when compared with nonsyndromic cases. Affected individuals often have thoracic deformities, with pectus carinatum upwards, and excavatum downwards; both deformities are capable of interfering with cardiac auscultation. A detailed cardiac evaluation, including electrocardiographic and echocardiographic examinations, is recommended for every patient with a diagnostic suspicion of Noonan syndrome.

This is a relatively frequent autosomal dominant inherited genic disease; the wide spectrum of its clinical manifestations or phenotype variability calls for the services of various specialists including pediatricians, endocrinologists, ophthalmologists, cardiologists, and hematologists. A better understanding of this heterogeneous syndrome may lead to more adequate follow-up and treatment of affected patients. 


\section{References}

1. Noonan JA, Ehmke DA. Associated noncardiac malformations in children with congenital heart disease. J Pediatr 1963; 63: 468-70.

2. Nora JJ, Nora AH, Sinha AK, Spangler RD, Lubs HA. The Ullrich-Noonan syndrome (Turner phenotype). Am J Dis Child 1974; 127: 48-55.

3. Allanson JE - Noonan syndrome. J Med Genet 1987; 24: 9-13.

4. Burch M, Sharland M, Shinebourne E, Smith G, Patton M, McKenna W. Cardiologic abnormalities in Noonan syndrome: phenotypic diagnosis and echocardiographic assessment of 118 patients. J Am Coll Cardiol 1993; 22: 1189-92.

5. Noonan J, O'Connor W. Noonan syndrome: a clinical description emphasizing the cardiac findings. Acta Pediatr Jpn 1996; 38: 76-83.

6. Jamieson CR, van der Burgt I, Brady AF, et al. Mapping a gene for Noonan syndrome to the long arm of chromosome 12. Nature Genet 1994; 8: 357-60.

7. van der Burgt I, Berends E, Lommen E, et al. Clinical and molecular studies in a large dutch family with Noonan syndrome. Am J Med Genet 1994; 53: 187-91.

8. Mendez HMM, Opitz JM. Noonan Syndrome: a review. Am J Med Genet 1985; 21: 493-506.

9. CharF, Rodriguez-Fernandez HL, Scott CI, Borgaonkar DS, Bell BB, Rowe RD. The Noonan syndrome-a clinical study of forty-five cases. BD: OAS 1972; VIII: $110-8$.

10. Ishizawa A, Oho SI, Dodo H, Katori T, Homma SI. Cardiovascular abnormalities in Noonan syndrome: the clinical findings and treatments. Acta Pediatr Jpn 1996; 38: 84-90.

11. Armengol AJ, Brohet CR, Intermans JP, Vliers A. Le ventricule gauche dans le syndrome de Noonan. Aspects électro-vecto-écho et angio-cardiographiques. Arch Mal Coeur 1987; 4: 445-53. 